Planetary Systems in the Universe - Observation, Formation and Evolution

Proceedings IAU Symposium No. 202, (C)2004 IAU

Alan Penny, Pawel Artymowicz, Anne-Marie Lagrange, \& Sara Russell, eds.

\title{
Modelling Planet Formation by Capture-Theory Interactions
}

\author{
Michael M. Woolfson and Stephen Oxley \\ Physics Department, University of York, York, YO10 5DD, UK
}

\begin{abstract}
Diffuse low-mass stars and brown dwarfs coexist with condensed solar-type stars in the embedded stage of a developing open cluster. It is shown by smoothed-particle-hydrodynamics modelling that interactions between stars and protostars leads to disruption of the protostar to form protoplanets that can then be captured by the star.
\end{abstract}

\section{Star Formation}

Observations of young stellar clusters, supported by theoretical results, show that the first stars produced in the parent dense cool cloud are of mass about $1.35 \mathrm{M}_{\odot}$ with progressively lesser mass stars produced as the cloud collapses (Williams \& Cremin 1969). Late in the star-formation process clusters reach a state of high stellar density in the range $10^{2}-10^{5} \mathrm{stars}^{\mathrm{pc}} \mathrm{c}^{-3}$, the so-called embedded stage (Lada \& Lada 1991). For example, in the core of the Trapezium cluster the density is $10^{4}-10^{5}$ stars $\mathrm{pc}^{-3}$ (McCaughrean \& Stauffer 1996). The duration of the embedded stage is $\sim 5 \times 10^{6}$ years during which the stars have a velocity dispersion $2-4 \mathrm{~km} \mathrm{~s}^{-1}$.

The lowest mass visible stars have masses about $0.075 \mathrm{M}_{\odot}$ but within recent years it has been possible to detect brown dwarfs (BDs) with masses below this limit. Thus Lucas \& Roche (2000) have detected 100 BDs in the Orion nebula plus 13 'free-floating planets' with masses in the range $8-13 \mathrm{M}_{\text {Jupiter }}$. Binney (1999) gives a mass function $f(M)=C M^{-\alpha}$ for BDs and most stars, with $\alpha=1.8$ for $0.01 \mathrm{M}_{\odot}<\mathrm{M}<2 \mathrm{M}_{\odot}$. With a BD lower limit of $0.013 \mathrm{M}_{\odot}$ this would give four times as many BDs as normal stars. Reid et al. (1999) suggest that there may be twice as many BDs as normal stars. Low-mass stars and BDs are produced late in the development of the cluster, when high cloud density gives a favourable Jeans critical mass. In the embedded stage there will be frequent interactions between condensed stars of around a solar mass and low-mass protostars and proto-BDs in a very diffuse state.

\section{The Capture Theory}

The Capture Theory (CT) for planet formation was first proposed by Woolfson (1964) and the validity of the basic mechanism has been confirmed since then by computational models of ever-increasing complexity. The CT involves a tidal interaction between a condensed star and a diffuse protostar in which a filament is drawn out of the protostar. Within this filament protoplanetary condensations 
form (Jeans 1919), and the protoplanets are captured by the condensed star. This model is free of all the objections raised against Jeans' original tidal theory. Its features are:

1. It is a dualistic theory and there are star-forming models available that give late-type stars with small angular momentum (e.g. Woolfson 1979).

2. The combination of filament density and temperature allows planetarymass condensations to form.

3. The angular momentum associated with the planetary orbits comes from that of the star-protostar orbit - there is no problem with planets as far out as Neptune.

4. The low temperature of planetary material allows light-element $(\mathrm{Li}, \mathrm{Be}$, B) abundance as observed on Earth - a difficultly for the Jeans theory.

5. The capture process leaves a resisting medium around the star with mass equal to several Jupiter masses.

6. For the solar-system scenario the resisting medium rounds off orbits on timescales $10^{6}$ years or less.

7. For the solar system, tidal interactions between the Sun and protoplanets give regular satellites.

8. The initial spin vector of the Sun has no special relationship to the mean plane of the system. Solid components of the resisting medium, drawn into the Sun by the Poynting-Robertson effect, pull the spin vector towards the normal to the mean plane giving the final $7^{\circ}$ tilt that is observed.

Observations of extra-solar planets show systems with characteristics very different from that in the solar system, in particular some having very close circular orbits and others orbits of high eccentricity. Any planet-forming process must allow variable parameters that could give rise to a wide variety of outcomes.

\section{Modelling a Star-BD Interaction}

We have developed a smoothed-particle-hydrodynamics code that includes realistic radiation transfer applicable to all opacities, optical depths and distributions of matter (Oxley 1999). This has been applied to simulating a tidal interaction between a solar-mass star and a proto-BD containing $\sim 11000 \mathrm{SPH}$ particles, with the following characteristics:

$\begin{array}{llll}\text { Mass of BD } & 0.05 \mathrm{M}_{\odot} & \text { Initial radius of BD } & 100 \mathrm{AU} \\ \text { Initial temperature of BD } & 25 \mathrm{~K} & \text { Star-BD orbit periastron } & 60 \mathrm{AU} \\ \text { Eccentricity of orbit } & 1.0 & \text { Initial star-BD separation } & 450 \mathrm{AU}\end{array}$

The outcome of the simulation is shown in Figure 1. In Figure 1(a) two protoplanets are seen to have formed. The lower one is captured but the upper escapes as a 'free planet'. The evolution of the captured protoplanet is illustrated in Figure 1(b) for the period 3000 to 4000 years from the beginning of the simulation. At the end of this period the compact core has mass $5 \mathrm{M}_{\text {Jupiter }}$ and it is surrounded by an extensive disk.

The inclusion of radiation is important. Removing the luminosity of the star, but retaining radiation transfer, leads to three captured protoplanets and three 'free planets'. 


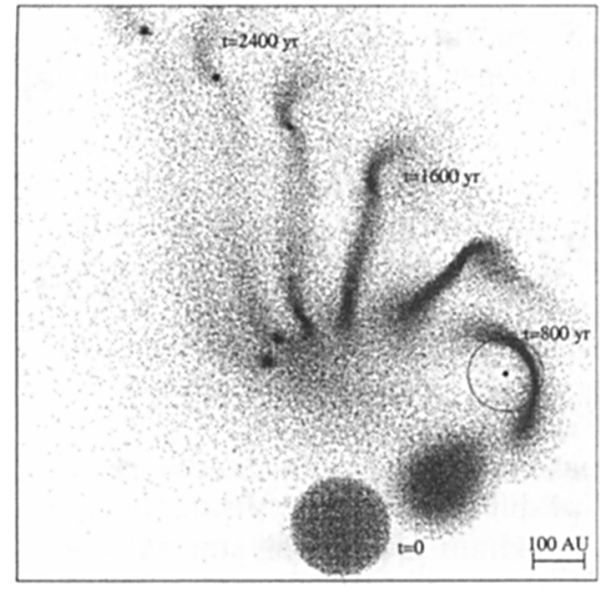

(a)

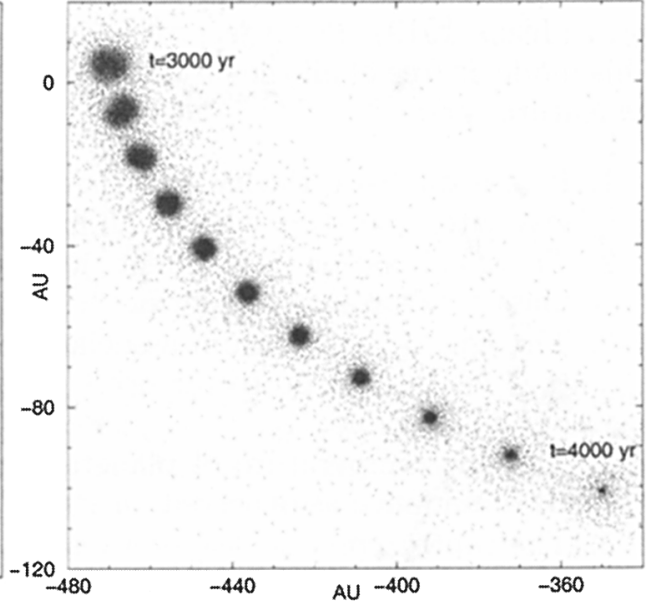

(b)

Figure 1. SPH modelling of an interaction between a solar mass star and a proto-BD. (a) Formation of a captured $(a=253 \mathrm{AU}$; $\mathrm{e}=0.86)$ and a 'free' protoplanet. (b) The condensation of the captured protoplanet.

\section{Conclusions}

The scale of the interaction modelled here is almost certainly too small and protostars or proto-BDs with radii $\sim 1000 \mathrm{AU}$ are more appropriate. The modelling can be scaled (dimension $\times \mathrm{k} \equiv$ time $\times \mathrm{k}^{3 / 2}$ ) but the scaling does not carry over to radiation transfer. With a larger scale system the probability of a disruption event can be estimated as several percent but the proportion of these leading to captured planets cannot be estimated at this time. However, if the assumptions of the model, and the modelling process itself, are valid then it seems almost certain that some, if not all planets are formed in this way.

\section{References}

Binney, J. 1999, MNRAS, 307, L27

Lada, C. J., \& Lada, E. A. 1991, in ASP Conf. Ser. Vol. 13, The Formation and Evolution of Star Clusters, ed. K. Janes (San Francisco: ASP), 3

Lucas, P. W., \& Roche, P. F. 2000, MNRAS, 314, 858

McCaughrean, M. J., \& Stauffer, J. R. 1996, AJ, 111, 1977

Oxley, S. 1999, D. Phil. thesis, University of York, www.droxley.freeserve.co.uk

Reid, I. N., Kirkpatrick, J. D., Liebert, J., Burrows, A., Gizis, J. E., Burgasser, A., Dahn, C. C., Monet, D., Cutri, R., Beichman, C. A., \& Skrutskie, M. 1999, ApJ, 521,613

Williams, I. P., \& Cremin, A. W. 1969, MNRAS, 144, 359

Woolfson, M. M. 1964, Proc. R. Soc., A282, 485

Woolfson, M. M. 1979, Phil. Trans. R. Soc., A291, 219 